\title{
Topological Excitation in Skyrme Theory
}

\author{
DUAN Yi-Shi, ZHANG Xin-Hui.* and LIU Yu-Xiao \\ Institute of Theoretical Physics, Lanzhou University, Lanzhou 730000, China
}

\begin{abstract}
Based on the $\phi$-mapping topological current theory and the decomposition of gauge potential theory, we investigate knotted vortex lines and monopoles in Skyrme theory and simply discuss the branch processes (splitting, merging and intersection) during the evolution of the monopoles.
\end{abstract}

PACS numbers: 12.39.Dc, 11.27.+d, 21.60.Fw

Key words: Skyrme theory, Knots, Monopoles

${ }^{*}$ Corresponding author; Electronic address: zhangxingh03@st.lzu.edu.cn 


\section{INTRODUCTION}

The Skyrme theory [1] has played an important role in physics, in particular in nuclear physics as a successful effective field theory of strong interaction. Witten [2, 3] revived this idea and proposed that baryons may be described phenomenologically as the topological solitons, which by now are called skyrmions. In this view the skyrmions are interpreted as the baryons and much evidence has been put forward [4, 5, 6]. Meanwhile, it has been discovered that the theory allows not only the topological skyrmions but also knots and monopoles [7]. Similar knots have appeared almost everywhere recently, in atomic physics in two-component Bose-Einstein condensates [8], in condensed matter physics in multi-gap superconductors [9] even in plasma physics in coronal loops [10]. Recently Ref. [7] suggested that the Skyrme theory is just a theory of self-interacting monopoles. We can see that a remarkable feature of the Skyrme theory is its rich topological structure. So one should pay much attention to the topological characteristic of the Skyrme theory.

In this paper, using the $\phi$-mapping topological current theory [11, 12] and the decomposition of gauge potential theory [13, 14], we research the inner topological structure of knots and monopoles in Skyrme theory. If we write the field $U$ in $\mathrm{SU}(2)$ space in a general form

$$
\begin{aligned}
& U=e^{i n F(\varphi)}=\cos F+i n \sin F, \\
& n=n^{a} \sigma_{a}, \quad n^{a}=\frac{\phi^{a}}{\|\phi\|},
\end{aligned}
$$

where $\phi^{a}(a=1,2,3)$ are three fundamental functions with space-time coordinates $x^{\mu}$ and $\sigma^{a}$ are pauli matrices, the Skyrme model is denoted by the Lagrangian [7]

$$
L=\frac{u^{2}}{4} \operatorname{Tr}\left(U^{+} \partial_{\mu} U\right)^{2}+\frac{\alpha}{32} \operatorname{Tr}\left(\left[U^{+} \partial_{\mu} U, U^{+} \partial_{\nu} U\right]\right)^{2}
$$

where $u$ and $\alpha$ are the coupling constants. It can be seen that the Lagrangian has a local $\mathrm{U}(1)$ symmetry as well as a global SU(2) symmetry [15]. Restricting $\varphi$ to $\pi$, one can reduce Eq. (3) to the Skyrme-Faddeev Lagrangian

$$
L_{s f}=-\frac{u^{2}}{2}\left(\partial_{\mu} \hat{n}\right)^{2}-\frac{\alpha}{4}\left(\partial_{\mu} \hat{n} \times \partial_{\nu} \hat{n}\right)^{2}
$$

whose equation of motion is given by:

$$
\hat{n} \times \partial^{2} \hat{n}+\frac{\alpha}{u^{2}}\left(\partial_{\mu} H_{\mu \nu}\right) \partial_{\nu} \hat{n}=0
$$


Here the tensor $H_{\mu \nu}$ is denoted by

$$
H_{\mu \nu}=\hat{n} \cdot\left(\partial_{\mu} \hat{n} \times \partial_{\nu} \hat{n}\right)=\epsilon_{a b c} n^{a} \partial_{\mu} n^{b} \partial_{\nu} n^{c}, \quad(a, b, c=1,2,3)
$$

which is a topological term describing the non-uniform distribution of the unit vector $\hat{n}$ at large distances in space. We will explore the topological properties of the Skyrme theory by analyzing the tensor $H_{\mu \nu}$.

The paper is arranged as follows. In Sec. III, using the $\phi$-mapping topological current theory and the decomposition of $U(1)$ gauge potential theory, we research the vortex lines in the Skyrme theory and knotted vortex excitation. The inner topological structure and the evolution of the monopoles will be studied in Sec. III. The conclusion of this paper is given in Sec. IV]

\section{KNOTTED VORTEX EXCITATION IN SKYRME THEORY}

In this section, based on the $\phi$-mapping topological current theory, we show that there is a two-dimensional topological current, which can be derived from $H_{\mu \nu}$, and the vortex lines in the skyrme theory are just inhering in this topological current. One can prove that the tensor $H_{\mu \nu}$ can be reexpressed as [16, 17]

$$
H_{\mu \nu}=\partial_{\mu} b_{\nu}-\partial_{\nu} b_{\mu}
$$

where $b_{\mu}$ is the Wu-Yang potential [18]

$$
b_{\mu}=\vec{e}_{1} \cdot \partial_{\mu} \vec{e}_{2}
$$

in which $\overrightarrow{e_{1}}$ and $\overrightarrow{e_{2}}$ are two perpendicular unit vectors normal to $\vec{n}$, and $\left(\overrightarrow{e_{1}}, \overrightarrow{e_{2}}, \vec{n}\right)$ forms an orthogonal frame:

$$
\vec{n}=\overrightarrow{e_{1}} \times \overrightarrow{e_{2}}, \quad \overrightarrow{e_{1}} \cdot \overrightarrow{e_{2}}=0
$$

Now, consider a two-component vector field $\vec{\phi}=\left(\phi^{1}, \phi^{2}\right)$ residing in the plane formed by $\vec{e}_{1}$ and $\overrightarrow{e_{2}}$ :

$$
e_{1}^{a}=\frac{\phi^{a}}{\|\phi\|}, \quad e_{2}^{a}=\epsilon_{a b} \frac{\phi^{b}}{\|\phi\|}, \quad\left(\|\phi\|^{2}=\phi^{a} \phi^{a} ; a, b=1,2\right) .
$$

It can be proved that the expression Eq. (10) for $\vec{e}_{1}$ and $\vec{e}_{2}$ satisfies the restriction Eq. (9). Obviously the zero points of $\vec{\phi}$ are just the singular points of $\vec{e}_{1}$ and $\vec{e}_{2}$. Using the $\vec{\phi}$ field, 
the Wu-Yang potential Eq. (8) can be written as

$$
b_{\mu}=\epsilon_{a b} \frac{\phi^{a}}{\|\phi\|} \partial_{\mu} \frac{\phi^{b}}{\|\phi\|} .
$$

Comparing the above formula with the decomposition of $U(1)$ gauge potential theory [13], we can see that Eq. (11) is just the expression of the $U(1)$ gauge potential decomposition, $b_{\mu}$ satisfies the $U(1)$ gauge transformation. Then the tensor $H_{\mu \nu}$ is reformed in terms of $\vec{\phi}(x)$

$$
H_{\mu \nu}=2 \epsilon_{a b} \partial_{\mu} \frac{\phi^{a}}{\|\phi\|} \partial_{\nu} \frac{\phi^{b}}{\|\phi\|} .
$$

According to Ref. [12], using $\partial_{\mu} \frac{\phi^{a}}{\|\phi\|}=\phi^{a} \partial_{\mu} \frac{1}{\|\phi\|}+\frac{\partial_{\mu} \phi}{\|\phi\|}$ and the Green function relation in $\phi$-space: $\partial_{a} \partial_{a} \ln \|\phi\|=2 \pi \delta(\vec{\phi})\left(\partial_{a}=\partial / \partial \phi^{a}\right)$, it can be proved that

$$
\frac{1}{4 \pi} \epsilon^{\mu \nu \lambda \rho} \epsilon_{a b} \partial_{\lambda} \frac{\phi^{a}}{\|\phi\|} \partial_{\rho} \frac{\phi^{b}}{\|\phi\|}=\delta^{2}(\vec{\phi}) D^{\mu \nu}\left(\frac{\phi}{x}\right),
$$

where $D^{\mu \nu}\left(\frac{\phi}{x}\right)=\frac{1}{2} \epsilon^{\mu \nu \lambda \rho} \epsilon_{a b} \partial_{\lambda} \phi^{a} \partial_{\rho} \phi^{b}$. Indeed if we introduce $\widetilde{H}^{\mu \nu}=\frac{1}{8 \pi} \epsilon^{\mu \nu \lambda \rho} H_{\lambda \rho}$, then we get $\widetilde{H}^{\mu \nu}=\delta^{2}(\vec{\phi}) D^{\mu \nu}\left(\frac{\phi}{x}\right)$. Defining the spacial components of $\widetilde{H}^{\mu \nu}$ as

$$
H^{i}=\widetilde{H}^{0 i}=\delta^{2}(\vec{\phi}) D^{i}\left(\frac{\phi}{x}\right), \quad(i=1,2,3)
$$

where $D^{i}(\phi / x)=D^{0 i}(\phi / x)$. From Eq. (14), we can see that $H^{i}$ exists if and only if $\vec{\phi}=0$. So it is necessary to study the zero points of $\vec{\phi}$ to determine the nonzero solutions of $H^{i}$. The implicit function theory [14] shows that under the regular condition

$$
D^{i}\left(\frac{\phi}{x}\right) \neq 0
$$

the general solutions of $\phi^{a}(\vec{x})=0$ represent the world surface of $N$ moving isolated singular strings $L_{k}(k=1,2 \cdots N)$ with string parameter $s$. These singular string solutions are just the vortex lines in the Skyrme theory. In $\delta$-function theory [19], one can prove that in three-dimensional space

$$
\delta^{2}(\vec{\phi})=\sum_{k=1}^{N} \beta_{k} \int_{L_{k}} \frac{\delta^{3}\left(\vec{x}-\vec{x}_{k}(s)\right)}{\left|D\left(\frac{\phi}{u}\right)\right|_{\Sigma_{k}}} d s,
$$

where $D\left(\frac{\phi}{u}\right)=\frac{1}{2} \epsilon^{j k} \epsilon_{m n}\left(\partial \phi^{m} / \partial u^{j}\right)\left(\partial \phi^{n} / \partial u^{k}\right)$ and $\Sigma_{k}$ is the $k$-th planar element transverse to $L_{k}$ with local coordinates $\left(u^{1}, u^{2}\right)$. The positive integer $\beta_{k}$ is the Hopf index of $\phi$-mapping, which means that when $\vec{x}$ covers the neighborhood of the zero point $\vec{x}_{k}(s)$ once, the vector 
field $\vec{\phi}$ covers the corresponding region in $\phi$ space $\beta_{k}$ times. Meanwhile the direction vector of $L_{k}$ is given by [12]

$$
\left.\frac{d x^{i}}{d s}\right|_{\vec{x}_{k}}=\left.\frac{D^{i}\left(\frac{\phi}{x}\right)}{D\left(\frac{\phi}{u}\right)}\right|_{\vec{x}_{k}} .
$$

Then considering Eqs. (16) and (17), we obtain the inner structure of $H^{i}$

$$
H^{i}=\delta^{2}(\vec{\phi}) D^{i}\left(\frac{\phi}{x}\right)=\sum_{k=1}^{N} \beta_{k} \eta_{k} \int_{L_{k}} \frac{d x^{i}}{d s} \delta^{3}\left(\vec{x}-\vec{x}_{k}(s)\right) d s,
$$

where $\eta_{k}=\operatorname{sgn} D\left(\frac{\phi}{u}\right)= \pm 1$ is the Brouwer degree of $\phi$-mapping, with $\eta_{k}=1$ corresponding to the vortex and $\eta=-1$ corresponding to the antivortex. From Eq. (18) one can obtain the topological number of vortex line $L_{k}$

$$
Q_{k}=\int_{\Sigma_{k}} H^{i} d \sigma_{i}=W_{k}
$$

in which $W_{k}=\beta_{k} \eta_{k}$ is the winding number of $\vec{\phi}$ around $L_{k}$.

Now we discuss the topological property of knotted vortex lines in the Skyrme system. First define a action, which is based on the so-called Hopf invariant

$$
I=\frac{1}{16 \pi^{2}} \int \epsilon^{i j k} b_{i} H_{j k} d^{3} x=\frac{1}{2 \pi} \int b_{i} H^{i} d^{3} x,
$$

where $i ; j ; k=1 ; 2 ; 3$ denote the 3-dimensional space. Substituting Eq. (18) into Eq. (20), we get

$$
I=\frac{1}{2 \pi} \sum_{k=1}^{N} W_{k} \int_{L_{k}} b_{i} d x^{i} .
$$

It can be seen that when these $N$ vortex lines are $N$ closed curves, i.e., a family of $N$ knots $\gamma_{k}(k=1, \cdots, N)$, then Eq. (21) leads to

$$
I=\frac{1}{2 \pi} \sum_{k=1}^{N} W_{k} \oint_{\gamma_{k}} b_{i} d x^{i} .
$$

This is a very important expression. Consider the $U(1)$ gauge transformation of $b_{\mu}: b_{\mu}^{\prime}=$ $b_{\mu}+\partial_{i} \theta$, where $\theta \in \mathbb{R}$ is a phase factor denoting the $U(1)$ transformation. It is seen that the $\partial_{i} \theta$ term in Eq. (22) contributes noting to the integral $I$, hence the expression (22) is invariant under the $U(1)$ gauge transformation. Meanwhile we know that $I$ is independent of the metric $g_{\mu \nu}$ [13]. Therefore one can conclude that $I$ is a topological invariant for the knotted vortex lines (a knot invariant) in the Skyrme theory. 


\section{MONOPOLES IN SKYRME THEORY}

In this section, we will explore the inner topological structure of monopoles in Skyrme theory by using the $\phi$-mapping topological theory. The generalized winding number $W$ can be obtained by integrating on a closed surface $\partial \Omega$, where $\Omega$ is a spacial volume and $\partial \Omega$ is its boundary. $W$ is defined by the Gauss map $n: \partial \Omega \rightarrow S^{2}[16]$

$$
W=\frac{1}{8 \pi} \int_{\partial \Omega} n^{*}\left(\epsilon_{a b c} n^{a} d n^{b} \wedge d n^{c}\right)
$$

where $n^{*}$ is the pullback of the map $n$. Then we have

$$
\begin{aligned}
W & =\frac{1}{8 \pi} \int_{\partial \Omega} \epsilon_{a b c} n^{a} \partial_{i} n^{b} \partial_{j} n^{c} d x^{i} \wedge d x^{j} \\
& =\frac{1}{8 \pi} \int_{\partial \Omega} H_{i j} d x^{i} \wedge d x^{j} \quad(i, j=1,2,3) .
\end{aligned}
$$

In topology this means that when $\vec{x}$ covers $\partial \Omega$ in the real space once, the unit vector $\hat{n}$ will cover $S^{2} W$ times. This is a topological invariant and is called the degree of Gauss map. At the same time, $W$ is also the total topological charge of the point defects (i.e. the singular points of $\hat{n}$ ) located in the volume $\Omega$. Using Stokes' theorem, we have

$$
W=\frac{1}{8 \pi} \int_{\Omega} \epsilon^{i j k} \epsilon_{a b c} \partial_{i} n^{a} \partial_{j} n^{b} \partial_{k} n^{c} d^{3} x=\int_{\Omega} \rho d^{3} x,
$$

where the density of point defects is derived from $H_{i j}$,

$$
\rho=\frac{1}{8 \pi} \epsilon^{i j k} \epsilon_{a b c} \partial_{i} n^{a} \partial_{j} n^{b} \partial_{k} n^{c}=\frac{1}{8 \pi} \epsilon^{i j k} \partial_{i} H_{j k}
$$

According to the $\phi$-mapping theory, the topological current is defined as

$$
J^{\mu}=\frac{1}{8 \pi} \epsilon^{\mu \nu \lambda \rho} \epsilon_{a b c} \partial_{\nu} n^{a} \partial_{\lambda} n^{b} \partial_{\rho} n^{c}, \quad(\mu=0,1,2,3),
$$

obviously, $\rho$ is the 0 -th component of $J^{\mu}$. Taking account of Eq. (2) and using $\partial_{\mu} n^{a}=$ $\phi^{a} \partial_{\mu}(1 /\|\phi\|)+(1 /\|\phi\|) \partial_{\mu} \phi^{a}, J^{\mu}$ can be expressed as

$$
J^{\mu}=\frac{1}{8 \pi} \epsilon^{\mu \nu \lambda \rho} \epsilon_{a b c} \frac{\partial}{\partial \phi^{l}} \frac{\partial}{\partial \phi^{a}} \ln (\|\phi\|) \partial_{\nu} \phi^{l} \partial_{\lambda} \phi^{b} \partial_{\rho} \phi^{c} .
$$

If we define Jacobian $\epsilon^{l b c} D^{\mu}\left(\frac{\phi}{x}\right)=\epsilon^{\mu \nu \lambda \rho} \partial_{\nu} \phi^{l} \partial_{\lambda} \phi^{b} \partial_{\rho} \phi^{c}$, and make use of the Green function relation in $\phi$ space [14], we obtain the $\delta$-function like current

$$
J^{\mu}=\delta(\vec{\phi}) D^{\mu}\left(\frac{\phi}{x}\right)
$$


The expression of Eq. (29) provides an important conclusion:

$$
J^{\mu}\left\{\begin{array}{l}
=0, \text { if and only if } \vec{\phi} \neq 0 . \\
\neq 0, \text { if and only if } \vec{\phi}=0 .
\end{array}\right.
$$

So it is necessary to study the zero points of $\vec{\phi}$ to determine the nonzero solutions of $J^{\mu}$. The implicit function theory [20] shows that under the regular condition

$$
D^{\mu}\left(\frac{\phi}{x}\right) \neq 0
$$

the general solutions of

$$
\begin{aligned}
& \phi^{1}\left(x^{1}, x^{2}, x^{3}, t\right)=0 \\
& \phi^{2}\left(x^{1}, x^{2}, x^{3}, t\right)=0 \\
& \phi^{3}\left(x^{1}, x^{2}, x^{3}, t\right)=0
\end{aligned}
$$

can be expressed as

$$
x^{1}=x_{k}^{1}(t), \quad x^{2}=x_{k}^{2}(t), \quad x^{3}=x_{k}^{3}(t),
$$

which represent the world lines of $N$ moving isolated singular pointlike solutions. These singular solutions are just the monopoles in Skyrme theory.

Above we have used the regular condition $D^{\mu}\left(\frac{\phi}{x}\right) \neq 0$. When this condition fails, branch processes will occur. It is known the velocity of the $k$-th zero is determined by

$$
\frac{d x^{i}}{d t}=\left.\frac{D^{i}\left(\frac{\phi}{x}\right)}{D^{0}\left(\frac{\phi}{x}\right)}\right|_{x=\vec{x}_{k}}, \quad(i=1,2,3) .
$$

It is obvious that when $D^{0}\left(\frac{\phi}{x}\right)=0$, at the very point $\left(t^{*}, \vec{x}^{*}\right)$, the velocity

$$
\begin{aligned}
\frac{d x^{1}}{d t} & =\left.\frac{D^{1}\left(\frac{\phi}{x}\right)}{D^{0}\left(\frac{\phi}{x}\right)}\right|_{\left(t^{*}, x^{*}\right)}, \\
\frac{d x^{2}}{d t} & =\left.\frac{D^{2}\left(\frac{\phi}{x}\right)}{D^{0}\left(\frac{\phi}{x}\right)}\right|_{\left(t^{*}, x^{*}\right)}, \\
\frac{d x^{3}}{d t} & =\left.\frac{D^{3}\left(\frac{\phi}{x}\right)}{D^{0}\left(\frac{\phi}{x}\right)}\right|_{\left(t^{*}, x^{*}\right)},
\end{aligned}
$$

is not unique in the neighborhood of $\left(t^{*}, \vec{x}^{*}\right)$. This very point $\left(t^{*}, \vec{x}^{*}\right)$ is called the bifurcation point. Without loss of generality, we discuss only the branch of the velocity component 
$\left(d x^{1} / d t\right)$ at $\left(t^{*}, \vec{x}^{*}\right)$. It is known that the Taylor expansion of the solutions of Eq. (33) in the neighborhood of $\left(t^{*}, \vec{x}^{*}\right)$ can generally be expressed as [12, 14]

$$
A\left(x^{1}-x^{1 *}\right)^{2}+2 B\left(x^{1}-x^{1 *}\right)\left(t-t^{*}\right)+C\left(t-t^{*}\right)^{2}+\cdots=0,
$$

where $A, B$ and $C$ are three constants. Then the above Taylor expansion leads to

$$
A\left(\frac{d x^{1}}{d t}\right)^{2}+2 B \frac{d x^{1}}{d t}+C=0 \quad(A \neq 0) .
$$

The solutions of Eq. (37) give different motion directions of the zero point at the bifurcation point. There are two possible cases

Case 1. For $\triangle=4\left(B^{2}-A C\right)=0$, from Eq. (37), we get only one motion direction of the zero point at the bifurcation point: $\left.\left(d x^{1} / d t\right)\right|_{1,2}=-B / A$, which includes three sub-cases: (a) one monopole splits into two monopoles; (b) two monopoles merge into one monopole; (c) two monopoles tangentially intersect at the bifurcation point.

Case 2. For $\triangle=4\left(B^{2}-A C\right)>0$, from Eq. (37), we get two different motion directions of the zero point: $\left(d x^{1} / d t\right)=\left(-B \pm \sqrt{B^{2}-A C}\right) / A$. This is the intersection of two monopoles, which means that the two monopoles meet and then depart at the bifurcation point.

In both cases 1 and 2, the sum of the topological charges of final monopole(s) must be equal to that of the initial monopole(s) at the bifurcation point.

\section{CONCLUSION}

In this paper, using the decomposition of $U(1)$ gauge potential theory and the $\phi$-mapping topological current theory, the inner topological structure of knots and monopoles in Skyrme theory is researched in detail. In Sec. II, we revel there exist topological vortex lines in Faddeev-Skyrme model, mainly research the knotted vortex lines and obtain a knot invariant. In Sec. III starting with the definition of the winding number, which is a topological invariant for point defects, we investigate the inner topological structure of monopoles and discuss the branch processes (splitting, merging and intersection). The topological charges are preserved in the branch processes during the evolution of monopoles. 


\section{Acknowledgment}

This work was supported by the National Natural Science Foundation and the Doctor Education Fund of Educational Department of the People's Republic of China.

[1] T.H.R. Skyrme, Proc. Roy. Soc. (London) 260 (1961) 127; 262 (1961) 237; Nucl. Phys. 31 (1962) 556.

[2] E. Witten, Nucl. Phys. B 223 (1983) 422.

[3] E. Witten, Nucl. Phys. B 223 (1983) 433.

[4] I. Zahed and G. Brown, Phys. Rep. 142 (1986) 1.

[5] A. Jackson and M. Rho, Phy. Rev. Lett. 51 (1983) 751.

[6] G. Adkin, C. Nappi, and E. Witten, Nucl. Phys. B 228 (1983) 552.

[7] Y.M. Cho, Phys. Rev. Lett. 87 (2001) 252001.

[8] J. Ruostekoski and J. Anglin, Phys. Rev. Lett. 86 (2001) 3934; H.T.C. Stoof, E. Vliegen, and U. Al Khawaja, Phys. Rev. Lett. 87 (2001) 120407; R.A. Battye, N.R. Cooper, and P.A. Sutcliffe, Phys. Rev. Lett. 88 (2002) 080401; C.M. Savage and J. Ruostekoski, Phys. Rev. Lett. 91 (2003) 010403.

[9] E. Babaev, Phys. Rev. Lett. 88 (2002) 177002; E. Babaev, L. Faddeev, and A. Niemi, Phys. Rev. B 65 (2002) 100512.

[10] L. Faddeev and A. Niemi, Phys. Rev. Lett. 85 (2000) 3416.

[11] Y.S. Duan, X. Liu, and P.M. Zhang, J. Phys: Condens. Matter 14 (2002) 7941.

[12] Y.S. Duan, H. Zhang, and S. Li, Phys. Rev. B 58 (1998) 125; Y.S. Duan and H. Zhang, Eur. Phys. J. D 5 (1999) 47; Y.S. Duan, X. Liu, and P.M. Zhang, J. Phys. A 36 (2003) 563.

[13] Y.S. Duan and X. Liu, Phys. Rev. D 67 (2003) 085002.

[14] L.B. Fu, Y.S. Duan, and H. Zhang, Phys. Rev. D 61 (2000) 045004.

[15] Y.M. Cho, Phys. Rev. Lett. 44 (1980) 001115; Phys. Lett. B 115 (1982) 125; Y.M. Cho and D. Maison, Phys. Lett. B 391 (1997) 360.

[16] Y.S. Duan and M.L. Ge, Sci. Sin. 11 (1979) 1072; Y.S. Duan and X.H. Meng, J. Math. Phys. 34 (1993) 1149.

[17] E. Fradkin, Field Theories of Condensed Matter Systems, ( Addison-Wesley, New York, 1991). 
[18] T.T. Wu and C.N. Yang, Phys. Rev. D 12 (1975) 3845; T.T. Wu and C.N. Yang, Phys. Rev. D 14 (1975) 437.

[19] J. A. Schouten, Tensor Analysis for Physicists (Clarendon, Oxford, 1951).

[20] É. Goursat, A Course in Mathematical Analysis, translated by E. R. Hedrick ( Dover, New York, 1904), Vol. I. 